\title{
A new scale for the assessment of competences in Cognitive and Behavioural Therapy
}

\author{
Anthony D. Roth \\ University College London, UK
}

\begin{abstract}
Background: Scales for assessing competence in CBT make an important contribution to research and practise. Aims: To develop a novel scale. Method: A new structured assessment tool is described which draws on a widely-used CBT competence framework to identify relevant areas of clinical practise. Results: Scale content was clarified through piloting and review by a range of stakeholders. Conclusion: Pending formal testing of the psychometric properties, the scale is ready for use to assess competences in cognitive and behavioural therapy.
\end{abstract}

\section{Introduction}

Determining how CBT is delivered is an important aspect of practice. For example, researchers need to identify fidelity to the treatment model in a clinical trial, training courses need to appraise the acquisition of skills in their students, and supervisors need some way of monitoring the development and maintenance of competence. To achieve this we need structured observation scales. To date a limited number of instruments have been developed, of which two are commonly applied. The first is the Cognitive Therapy Rating Scale (CTRS) in its original (Young \& Beck, 1988) and revised forms (e.g. the Cognitive Therapy ScaleRevised (CTS-R): Blackburn, James, Milne, Baker, Standart, Garland \& Reichelt, 2001); the second is the CBT subscale of the Collaborative Study Psychotherapy Rating Scale (CSPRS: Hollon, Evans, Auerbach, DeRubeis, Elkin, Lowery, Kriss, Grove, Thason \& Piasecki, 
1988). While use of the latter scale seems to be largely restricted to research trials, the CTRS/CTS-R is widely employed by researchers, trainers and clinicians.

This paper introduces a new scale for structured observation of $\mathrm{CBT}^{1}$. Although the development of a new scale is not dependent on deficiencies in prior ones, the CTRS/CTS-R is problematic in at least three areas. Firstly, although groups of raters working together can achieve high levels of concordance, the reliability of ratings by independent judges is uncertain (e.g. Reichelt, James \& Blackburn, 2003). Secondly, the scale subsumes the use of change techniques under one item, though this is an area of competence where there is much to be said for specificity. Thirdly, since the inception of the scale it has become clear that CBT needs to be adapted when working with different conditions (particularly the anxiety disorders), making the generic nature of the CTS increasingly problematic ${ }^{2}$.

\section{UCL scale for structured observation of CBT}

The UCL scale is rooted in the competence framework for CBT (Roth \& Pilling, 2007), developed as part of the English IAPT programme and used to generate the IAPT CBT curriculum for people presenting with anxiety and depression. The framework organises the delivery of CBT into discrete areas of activity, and identifies the knowledge and skills that underpin all variants of CBT as well as specific CBT skills that are applied when working with specific conditions or presentations.

\footnotetext{
${ }^{1}$ The scale and associated background documents are available from the author

${ }^{2}$ Several client or disorder-specific adaptations of the CTS-R have also been developed to capture the full range of CBT techniques applied when working with different populations - for example for people with social anxiety (Von Consbruch, Clark, \& Stangier, 2012), or for people with psychosis (Haddock, Devane, Bradshaw, McGovern, Tarrier, Kinderman, Baguley, Lancashire \& Harris, 2001).
} 
The framework also includes a domain of Generic Therapeutic Competences, areas of knowledge and skills that are common across therapy modalities (for example, relational competences (such as alliance building and repair)) and skills associated with the management of sessions (for example, using measures, responding to emotional expression, or ending sessions). Although generic competences are necessary skills for the effective delivery of therapy, it is helpful to separate them from CBT-specific skills. As such two parallel scales were developed, both of which would usually be administered, focusing on generic and CBT competences respectively.

\section{Scale development}

The CBT competence framework includes a 'map' setting out the discrete areas of clinical activity that constitute CBT practice, and this was used to identify a set of items for the scale. This initial set was modified on the basis of feedback from clinicians piloting early versions of the scale; as shown in Table 1 the resulting set of 26 items is organised into four sections:

\section{Underpinning CBT techniques}

2. Change techniques based on discussion and experiential methods

3. Change techniques deployed for specific conditions

4. Reviewing the session as whole

A similar procedure was used to develop a 13-item 'Core and Generic Therapeutic Competences' scale; these are set out in Table 2. The grouping of this table broadly reflects the structure of the competence framework, with a final section intended to provide a 'helicopter' view of the session as a whole. 
Each item is anchored with synoptic behaviourally-specific descriptions that indicate what an observer would expect to see if the competence were being performed appropriately (with these descriptions drawn from those contained in the framework). The descriptors are set at the level that an expert practitioner would demonstrate, and to which a trainee therapist would aspire. By way of illustration, Table 3 shows the behavioural anchors for two items: 'agenda setting and structuring sessions' (one of the underpinning CBT techniques), and 'implementing CBT using a collaborative approach' (found in the section 'reviewing the session as a whole'). These anchors can include multiple concepts, reflecting the fact that the activity they describe is exemplified by a set of actions that (even if different in their focus) are coherent in their intent: all need to be present for the item to be rated as competently delivered. For example, however well an agenda is set, the session needs to be structured in a way that enables this agenda to be realised; one activity without the other is unhelpful.

\section{Rating}

Ratings are made on a five-point Likert scale, with each point linked to anchors which specify both the extent to which a skill is present and the degree to which it needs to be developed., as shown in Table 4. A short guide to the generic and CBT scales sets-out their rationale and the way in which they are rated and scored, and directs raters to the CBT framework for a fuller description of the competences.

Most competence scales require raters to score all the items, but this can be problematic if areas of activity are not relevant to a specific intervention package or to a specific session. This observation is particularly pertinent to the present scale because of the detail with which it specifies change mechanisms. As such, raters have the option of marking an area of activity as 'not-applicable' when it is absent from the session, but its absence is expected or is 
justified by the context. For example, 'working with imagery' could be rated 'non-applicable' if imagery did not feature in a session, but if a client described obviously relevant imagery which the therapist did not address then the rater would score this as an area needing development. Clearly judgment is needed to decide whether - in the context of the session an area of activity is appropriately absent, or whether its absence should flag a concern.

\section{Feedback on the use of the scales}

Detailed feedback on the scales has been obtained through several routes - iterative review from senior CBT practitioners who commented on the scales at different points in their development, piloting of the generic and CBT scales by trainee clinical psychologists and their supervisors, feedback from raters conducting a pilot study of the scale's reliability, and feedback from raters in the preparatory stages of an appropriately-powered inter-rater reliability study. This process of iterative feedback has been used to make improvements to both the scale and the rating system.

\section{Psychometric properties of the scale and Inter-rater reliability}

Understanding the psychometric properties of the scale is the next stage in its development, with inter-rater reliability a primary and critical consideration if it is to be used to make meaningful summative judgments. This is especially important in relation to setting a cutpoint for competence, which at present is represented qualitatively (in that a score of 3 or more indicates that the competence is demonstrated, but to varying degrees of proficiency). An ongoing study is exploring these issues, with a fully-crossed design in which six independent and experienced judges rate the same 30 recordings on both the UCL scale and the CTS-R. This should afford a detailed picture of the properties of the scale and yield information on the performance of individual items within it. 


\section{References}

Blackburn, I.M., James, I.A., Milne, D.L., Baker, C., Standart, S., Garland, A. \& Reichelt F.K. (2001). The revised cognitive therapy scale (CTS-R): psychometric properties. Behavioural and Cognitive Psychotherapy, 29, 431-446. DOI: 10.1017/S1352465801004040

Haddock, G., Devane, S., Bradshaw, T., McGovern, J., Tarrier, N., Kinderman, P., Baguley, I., Lancashire, S. \& Harris, N. (2001). An investigation into the psychometric properties of the Cognitive Therapy Scale for Psychosis (CTS-Psy). Behavioural and Cognitive Psychotherapy, 29, 221-233. DOI: 10.1017/S1352465801002089

Hollon, S. D., Evans, M. D., Auerbach, A., DeRubeis, R. J., Elkin, I., Lowery, A., Kriss, M., Grove, W., Thason, V. B. \& Piasecki, J. (1988). Development of a system for rating therapies for depression: Differentiating cognitive therapy, interpersonal therapy, and clinical management pharmacotherapy. (Unpublished manuscript, University of Minnesota, Twin Cities Campus.)

Reichelt, F. K., James, I. A. and Blackburn, I.-M. (2003). Impact of training on rating competence in cognitive therapy. Journal of Behavior Therapy and Experimental Psychiatry, 34, 87-99. DOI:10.1016/S0005-7916(03)00022-3

Roth, A. D., \& Pilling, S. (2007). The competences required to deliver effective cognitive and behavioural therapy for people with depression and with anxiety disorders. London: Department of Health. 
Von Consbruch, K., Clark, D.M., \& Stangier, U. (2012). Assessing therapeutic competence in cognitive therapy for social phobia: psychometric properties of the cognitive therapy competence scale for social phobia (CTCS-SP). Behavioural and Cognitive Psychotherapy, 40, 149-61. DOI: 10.1017/S1352465811000506

Young, J., \& Beck, A. (1988). Cognitive therapy scale. Unpublished Manuscript. 
Table 1

Structure of the Generic scale

\section{Structure of the Generic scale}

1. Establishing the professional context

2. Non-verbal behaviour

3. Working with difference

4. Structure and pacing

5. Active listening and empathy

6. Undertaking a generic assessment

7. Communicating a formulation/plan of intervention

8. Discussing the intervention plan

9. Responding to emotional content

10. Collaboration

11. Developing and fostering the therapeutic alliance

12. Management of threats to the alliance

13. Using measures

14. Ending sessions 
Table 2

Structure of the CBT scale

\section{Structure of the CBT scale}

\section{Section 1: Underpinning CBT techniques}

1. Structuring sessions and agenda setting

2. Using summaries and feedback

3. Guided discovery and Socratic questioning

4. Identifying maintenance cycles

5. Sharing a CBT formulation

\section{Section 2: Change techniques based on discussion and experiential methods}

\section{Using thought records}

7. Working with safety behaviours

8. Detecting, examining and helping clients reality test automatic thoughts and images

9. Identifying and modifying assumptions

10. Working with beliefs

11. Working with imagery

12. Planning and reviewing practice assignments ${ }^{3}$

13. Planning and conducting behavioural experiments

14. Activity monitoring and scheduling

15. Problem solving

16. Conducting exposure

17. Working with endings

\section{Section 3: Change techniques deployed for specific conditions}

18. Specific change techniques for working with panic

19. Specific change techniques for working with GAD

20. Specific change techniques for working with OCD

21. Specific change techniques for working with social anxiety

22. Specific change techniques for working with trauma

\section{Section 4: Reviewing the session as a whole}

23. Implementing CBT using a collaborative approach

24. Using measures

25. Using change techniques appropriate to the client's presentation and problems

26. Metacompetences

\footnotetext{
3 'The phrase practice assignments' is synonymous with the more traditional term 'homework', and is the term employed in the CBT competence framework
} 
Table 3

Examples of scale items and their behavioural anchors

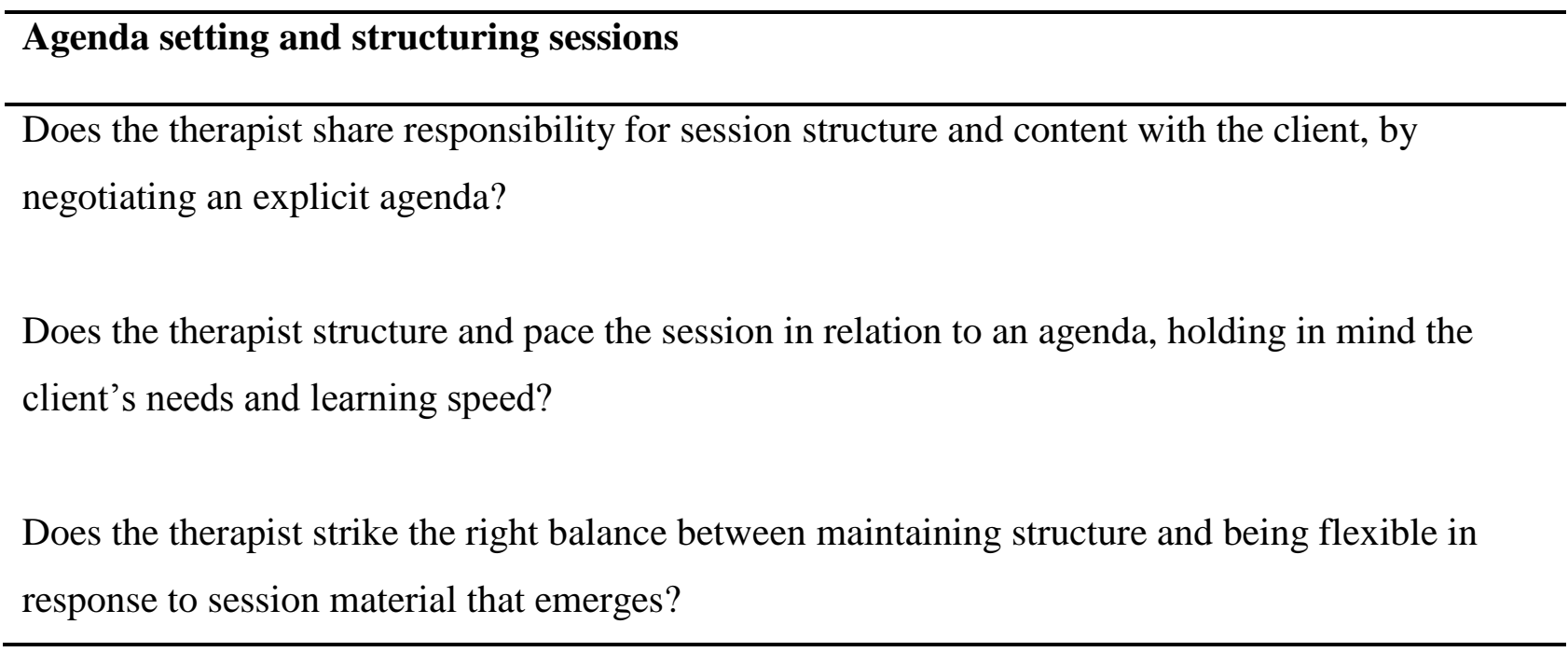

\section{Implementing CBT using a collaborative approach}

Did the therapist consistently foster collaborative working - encouraging the client to take an active role in the therapy, such that the client and therapist work as a "team"

Did the therapist consistently use activities that encourage the client to share responsibility for the therapy (e.g. inviting shared agenda setting, shared discussion, shared problem solving, shared decision-making, Socratic questioning etc.)

Did the therapist identify and discuss any difficulties clients have in working collaboratively and discuss these with the client in a manner congruent with the CBT model 
Table 4 Rating scale

\section{Competence not demonstrated or requires major development}

\begin{tabular}{|l|l|}
\cline { 2 - 3 } & Relevant technique or process is not present, but should be \\
\cline { 2 - 3 } & $\begin{array}{l}\text { Relevant technique or process is barely present and/or it is applied in a } \\
\text { manner that is ineffective* for this client }\end{array}$ \\
\hline \hline
\end{tabular}

Competence only partially and/or poorly demonstrated and requires significant development

Only some aspects of the relevant technique or process are apparent, and/or it is applied in a manner that is only marginally effective* for this client

\section{Competence demonstrated but requires further development}

Relevant technique or process is present but delivered in a manner that is partial and so not as effective* as it could be for this client, with a number of aspects requiring development (for example because it needs to be targeted more accurately to the client's presentation, or applied more consistently or coherently)

\section{Competence demonstrated well but requires some specific development}

\begin{tabular}{|c|c|c|}
\hline & $\begin{array}{l}\text { Relevant technique or process is applied well and delivered in a } \\
\text { manner that is effective* for this client's presentation; however there } \\
\text { are some specific (but not critical) areas for development }\end{array}$ & 4 \\
\hline \multicolumn{3}{|c|}{ Competence demonstrated very well and requires no substantive development } \\
\hline & $\begin{array}{l}\text { Relevant technique or process is applied fluently and coherently, in a } \\
\text { manner that is demonstrably effective* for this client }\end{array}$ & 5 \\
\hline
\end{tabular}

\section{Rating an item as 'not applicable'}

This rating is used if an area of activity is not present, AND (in the rater's view) does not need to be present because it is not relevant to, or required in, the specific session being rated.

If an area of activity is not present but (in the rater's view) it should be, then it should be rated as ' 1 ' (indicating that the competence was not demonstrated and should have been). 